\title{
Risks and Opportunities for Youth in the Digital Era
}

\author{
A Cyber-Developmental Approach to Mental Health \\ Vasileios Stavropoulos ${ }^{1,2} \odot$, Frosso Motti-Stefanidi ${ }^{1}$, and Mark D. Griffiths ${ }^{3}$
}

\begin{abstract}
Due to continued groundbreaking digital advancements, internet use has increased significantly. This has led a heated debate in relation to weighing the many advantages of the technology against its potentially deleterious effects. To address such questions, experts converge on the need for greater knowledge around the way individual differences, partly shaped by an individual's unique experiences of engaging with the medium, and partly by other real-life experiences, lead to different developmental trajectories. Consequently, the goals of the present review are to: (i) broadly describe differences in digital media applications, users and usage; (ii) introduce the Cyber-Developmental Framework (CDF), as an overarching framework for understanding individual differences in adaptive and maladaptive digital media use among youth; (iii) delineate the cyber-component of this framework in relation to users' experience of the digital context, their activity within it, as well as their digital self-presence, which may have an impact on their digital media use; and (iv) summarize priorities and future directions through the lens of this CDF. Within this context, this review particularly emphasizes the effect of digital media use on youth's psychological well-being. It is argued that the trajectory youth will follow in their use of the internet is a function of the interplay between their own characteristics, their proximate and distal contexts, and the particular features of the digital application(s) that the individual is engaged in. The review points to the need to conduct research focusing on better understanding the developmental and digital-context related influences on youth's trajectories of internet use.
\end{abstract}

Keywords: Internet use and abuse; Youth; Mental health; development; human-computer interaction, digital media 


\section{Risks and Opportunities for Youth in the Digital Era: A Cyber-Developmental Approach to Mental Health}

The 'digital' or 'third industrial revolution' initiated approximately 50 years ago, signifying a massive transition from mechanical and analogic communication to digital media (Schoenherr, 2004). These media include all forms of audiovisual, application-software and/or other types of content, which may be generated, modified, saved or sourced via a digital channel, using a code that encrypts and translates data (Smith, 2013). As the major volume of digital media employs the internet, the terms are often utilized interchangeably (Chayko, 2020). Due to the growth of such technologies, many define the most recent years as the beginning of the 'fourth industrial revolution', also known as the 'digital era' or the 'second coming' (Coldwell, 2019). This embraces the life changing innovations in information technology (IT), computermediated communication (CMC), and new media applications accommodated by the internet (Coldwell, 2019; PEW, 2020). Consequently, the term 'digital natives' emerged to describe those who have become familiar with new technologies early in life and distinguish them from 'digital immigrants', who were exposed to digital means in adulthood (Prensky, 2009).

The present review focuses on the impact of such changes on the development and the mental health particularly of digital natives. It is organized in four sections. Firstly, different digital media applications, users and usage behaviors will be briefly introduced. Secondly, theoretical milestones in understanding digital media use and abuse will be illustrated. Thirdly, a dialectical discourse between digital media and developmental science will be promoted via the introduction of a hybrid, integrative, Cyber-Developmental Framework (CDF). Fourthly, priorities and future directions will be highlighted.

\section{Digital media applications, users and usage behaviors}

Contemporary digital media accommodate a range of instrumental, socialization and entertainment aspects (Smith, 2013). Instrumental aspects involve uses dictated by an individual's real-life needs across life-domains such as education, employment and health (Gomes \& Dias, 2019). Socialization applications, broadly called social media, involve the generation and distribution of information, opinions, interests and ideas, using online communities and networks (Beyens, Pouwels, van Driel, Keijsers, \& Valkenburg, 2020). 
They occupy a range of areas including career and employment (e.g., LinkedIn), research (e.g., ResearchGate), romantic and/or sexual relationships (e.g., Tinder), friendships (e.g., Facebook), and even political activation (e.g., Twitter, Reddit; Kemp, 2019). Social media present with a 45\% level of global penetration, reaching 98\% among developed nations (Kemp, 2019).

Entertainment applications involve various digital portals providing individuals with amusement/enjoyment, such as digital games and audio-visual recordings (Chayko, 2020). Internet/digital games maybe enhanced by naturalistic animations and augmented reality features, which mix online and offline world (e. g. game objects in real locations, such as in videogames like Pokemon Go; Hamari, Malik, Koski \& Johri, 2019). Games can be played exclusively by one gamer or to allow multiple players (i.e., massively multiplayer online games [MMOs]). Some games require the development and customization of an in-game representation role/persona, known as the avatar (i.e., role playing games; [RPGs]). The latter might be also combined with the multiplayer feature resulting to massively multiplayer online role-playing games (MMORPGs; Jones, Scholes, Katsikitis, \& Carras, 2014). Most games converge on a levelled structure with progressively higher demands and rewards, while they may have different themes (e.g., action, real-life simulation, adventure, sports, racing, etc.) to appeal different audiences (Gough, 2020).

Furthermore, innovative gaming-type platforms integrating physical and/or social media activities emerge. The application STRAVA uses geocoded data to track an individual's cycling and running and report them on a multi-user portal. STRAVA allocates trophies to reward users' performance, cultivating competition, such that users' fitness motivation increases (Westlake, 2020). Similarly, the Virtual Reality [VR] Chat enables users to socialize with others via avatars, without entailing competition and/or challenges (e.g., going to virtual gatherings, situated within a virtual context via an avatar; Saffo, Yildirim, Di Bartolomeo, \& Dunne, 2020). Such features underpin the expansion of digital games, currently played by over 2.7 billion people (WePC, 2020). Alongside, other entertainment applications, such as virtual pornography, expand annually at over $10 \%$, with $25 \%$ of children between 7 to 12 years having accessed online porn on a monthly basis (Absolute Market Insights, 2020; Lewczuk, Wojcik, \& Gola, 2019). 
These differences are combined with geographical variations in digital media usage. The prevalence of digital media usage in Northern America and Northern Europe is 95\% compared to just 12\% in Middle Africa (Kemp, 2019). Geographical differences occur also regarding the types of digital media applications used. In Middle Africa just 7\% of the general population are social media users, compared to over $70 \%$ in North America (PEW, 2019). A digital gender divide has been additionally observed, with males using the internet $12 \%$ more than females, and such differences hiking to $25 \%$ in Africa (International Telecommunications Union, 2017). Gender differences expand to one's applications of use, with gaming being male dominated (15\% - 24\%; Gough, 2020). These internet use trends are skewed towards younger populations, with $91 \%$ of young individuals, vs. $71 \%$ of the general population, using the internet daily within the EU (Eurostat, 2020). Overall, digital natives appear to use social media (e.g., Instagram, 72\%; Snapchat, 69\%), digital games (84\%), internet forums (e.g., reddit, 7\%) and online video channels (e.g., YouTube, 85\%; PEW, 2018).

The aforementioned differences are combined with variability in use patterns. Abstinence from digital media, as well as moderate and excessive levels of use lie on a continuum, (Anderson, Steen \& Stavropoulos, 2017). Those who abstain from digital media use, either because they are lacking skills (e.g., digital illiteracy), or access, are likely to suffer lower employability, lower income and lower time resources (Hernandez \& Roberts, 2018). Moderate users, who may use the internet for their real-life needs appear to benefit across domains such as employment, relationships and health (Gomes \& Dias, 2019). In contrast, excessive users may find their lives being compromised by their internet absorbance (Stavropoulos, Kuss, Griffiths, Wilson \& Motti-Stefanidi, 2017). Interestingly, the rates of those who problematically use the internet appear to vary between $2.6 \%$ and $10.9 \%$, depending on the population examined and the definition adopted (Cheng \& Li, 2014).

Such differences coexist with variations considering digital media impact on one's psychological well-being and mental health (PEW, 2018). For instance, US teens report that social media helped them (i) connecting with family and friends $(40 \%)$, (ii) meeting others with similar interests (15\%), (iii) having 
easier access to information/news (16\%), (iv) entertaining (9\%), and (v) expressing themselves (7\%). The same adolescents though reported that social media had negatively impacted them via (i) bullying and rumor spreading (27\%), (ii) harming relationships (17\%), (iii) cultivating unrealistic views for life (15\%), (iv) causing addictions/distractions (14\%), and (v) enhancing peer pressure (12\%; PEW, 2018). Similarly, while gaming participation may produce positive emotions, enhance relationships when played in groups, and give a sense of meaning and achievement to youth (Jones et al., 2014), excessive gaming can significantly compromise gamers' health and wellbeing (Brand et al., 2020). Likewise, online pornography may promote the exploration of young people's sexual identity but can also accommodate the projection of idealized sexual expectations considering appearance and/or activity (Litsou et al., 2020).

\section{Theoretical milestones in understanding digital media use and abuse}

Different conceptualizations have been proposed to account for individual differences in the use of digital media. Initially researchers focused on explaining internet abuse, whereas more recent approaches focus on the full range of functioning, ranging from normative to problematic internet use, and examining it in social context.

The Cognitive-Behavioral Model for Pathological Internet Use (PIU; Davis, 2001), emphasizes excessive internet use. This may be generalized, referring to over-engagement in a range of internet applications, or specific, referring to exclusively one type of internet activity. As is the case with other problematic behaviors, pathological internet use is according to Davis (2001) the result of "who" the user is (e.g., pre-existing vulnerabilities, diathesis) and "what" they are experiencing in their lives (e.g., stress). The interaction between "who" and "what" is co-defined by distal and proximal individual-level factors. Distal factors may involve psychopathological tendencies, such as depression. Proximal factors entail maladaptive cognitions about the self and/or the world, which may become enhanced by cognitive distortions and ruminating processes (e. g. an individual recycling negative views of themselves and the future). 
Douglas et al. (2008) expanded Davis' (2001) work by conceptualizing problematic internet use as an addiction. They posited that an individual's interaction with digital media depends on factors that may 'push' and 'pull' them online. Push factors involve user characteristics, such as personality or psychological symptoms, as well as characteristics in their surroundings (e.g., peers and family), that may encourage online use. Pull factors are application features that may attract individuals online, such as the online environment and/or the way they experience themselves online. They noted that it is the interplay between push and pull factors that fuels negative outcomes, such as internet addiction symptoms, sleep disruption, and reduction in an individual's academic, relationship/interpersonal and occupational performance.

Brand and colleagues (2016) proposed the Interaction of Person-Affect-Cognition-Execution (IPACE) model, also considering problematic internet use as an addiction. In brief, their model, emphasizes the interaction between: (i) characteristics of users, such as their personality traits, psychopathological symptoms, cognitions and internet use motivations; and (ii) their way of thinking and feeling, such as their affective and cognitive responses to situationally induced distress. Examples of the latter include users' potentially impulsive coping strategies involving internet use, their biases/distorted expectations about internet, their cravings and urges for mood regulation, and their attentional biases. Finally, the model emphasizes how users process their choices. These involve an individual's inhibitions and decision-making steps while engaging with specific online applications. Therefore, they suggest that when an individual becomes addicted to a digital application, they initially do it to feel better (i.e., gratification/pleasure seeking) and later on just to feel less negatively (i.e., compensate for the negative feelings triggered by their previous addictive use).

However, it is important to understand both adaptive and maladaptive internet use. The Theoretical Framework for Children's Internet Use (TFCIU) adopts a developmental and contextualized approach to individual differences in internet use (Livingstone \& Haddon, 2012). This model is based on Bronfenbrenner and Morris' (2007) bioecological model of human development. Their conceptualization differs from the those of Davis (2001) and Brand et al. (2016) in that, first it explains the full range of 
diverse behaviors related to media use, instead of focusing solely on problematic use and second, it considers one's developmental context. They argue that to understand why some young people engage in normative use, whereas others engage in excessive use, researchers need to examine the role of their proximal social contexts, such as their family, peers and school, as well as the role of the societal and cultural distal context. The latter include the social, economic, and political landscape, the laws of the land, and the attitudes of its citizens towards issues such as globalization and digitalization. The proximal and distal social context effects are also expected to be bidirectional. The way young individuals use the internet may influence the functioning and response of their family, schools, and peers, as well as that of the society in which their lives are embedded (Livingstone \& Haddon, 2012). In 2018, Livingstone, Mascheroni, and Staksrud, expanded the TFCIU model by considering an "online inner box", which reflects risks and opportunities experienced by the child while online, as an additional effect (i.e., what is happening when the child is online). They also introduced the concept of "digital ecology" to describe the effects of the digital world itself on youth's internet use.

These appear to correlate with the notion of the "techno-subsystem" posited earlier by Johnson and Puplambou (2008). They also adopted Bronfebrenner and Morris' (2007) sequence of successively embedded layers of context, where in the interim space between the individual and their micro-system, (e. g. family and peers in one's immediate context) they situated a techno-subsystem. Any influences on the individual due to the use of all forms of digital technology devices, such as mobile phones and computers, were included in this layer/level. They argued that Bronfebrenner and Morris' (2007) did not differentiate the influences of media-technology, such as screen-time effects, from an individual's microsystem. Nonetheless, they supported that the complexity and the continuous advancements of digital media pose the need for the consideration of a separate subsystem. According to their proposal, the techno-subsystem involves the user's interplay with both living (e.g., peers) and artificial (e.g., hardware) elements of an individual's environment via the use of digital applications. Therefore, it constitutes a part of an individual's direct, and in some cases more private, than even their family and peers, surrounding. 
Valkenburg and Peter (2013) also adopted a developmental and contextualized approach to emphasize an age independent understanding of digital media effects. They proposed the Differential Susceptibility to Media Effects Model (DSMEM), which includes different influences on youth's internet use. The first key influence is users' potential susceptibility to media, which may be related to their values, attitudes, beliefs, motivations, and/or moods. The second influence is developmental, which refers to developmental changes in relation to cognitive, emotional and social behaviors potentially related to their maturation. They also stressed the role of the influence of youth's social context. As is the case in Livingstone's model (e.g., Livingstone \& Haddon, 2012), they refer to proximal level influences such as influence of the family, friends, peers, school, church, and work and to distal level influences, such as the influence of societal cultural norms and values. Valkenburg's and Peter's (2013) additionally suggested that the quality of digital media content, which may be related to specific response states (i.e., cognitions, emotions and arousal/excitative), requires to be further clarified. Their recommendation aligns with that of Livingstone et al. (2018), who called for a better understanding of the digital context/ecology itself, as well as an individual's unique experience within it.

\section{The Cyber-Developmental Framework: A Dialectical Discourse between Digital Media and Developmental Science}

To address these recommendations, we introduce a multi-level cyber-developmental framework (CDF), for understanding the diverse pathways into online technology use and what factors and processes may explain the diversity in outcomes. This model merges sources of influence from both the developmental and the digital media literature to delineate differences within and between individuals, as well as between groups. Therefore, the model aims to address questions such as "how do youth differ in the way they use the digital media?", "whether and how do youth's peer group, family and/or community contribute to individual differences in internet use?" "whether and how do digital media users change over time?", Next, concepts and principles of the cyber-developmental framework will be briefly presented. Firstly, key criteria for assessing digital media use outcomes, and, secondly, the role of individuals' own 
characteristics, as well as that of real-life and virtual-context influences, on individual differences in digital medial use will be examined in line with past literature (Odgers \& Jensen, 2020; Orben 2020; Stiglic \& Viner, 2019).

\section{Digital Media Use Outcomes}

The outcomes of digital media use should be viewed in relation to one's stage of development, especially during adolescence and emerging adulthood (Jackson \& Goossens, 2020). During this time, young people are faced with, and need to address, key developmental challenges. These have been organized in broad domains, such as individual development, relationships with parents, teachers, and peers, and functioning in the proximal environment and in the broader social world (Masten \& MottiStefanidi, 2020). Positive adaptation with respect to developmental tasks may be judged based on external behaviors. These include success in school, having close friends/being accepted by peers, exhibiting positive conduct, and civic engagement, as well as becoming increasingly independent and accountable. It can also be assessed in terms of internal adaptation, such as development of self-regulation, or a cohesive and integrated sense of personal identity (Masten \& Motti-Stefanidi, 2020). How well youth negotiate these challenges defines the quality of their adaptation and is linked to their mental health and psychological well-being. Youth may follow diverse pathways in their adaptation with respect to these developmental tasks as a result of complex interactions between the individual and their real and virtual contexts, in which their lives are embedded (Masten \& Motti-Stefanidi, 2020).

The present model acknowledges that while digital media technology may be aiming for adaptive digital media usage, it also has the potential to place at risk youth's adaptation, as a result of the interplay between user, application, and real-life as well as virtual-context factors. Consequently, it defines as adaptive all forms of digital media use that promote individuals' achievement of developmental tasks as well as their mental health, while it defines as maladaptive all forms that compromise them (Masten \& Motti-Stefanidi, 2020).

\section{Adaptive outcomes related to digital media use}


Digital competence assumes a sufficient level of awareness/usability of the opportunities and risks entangled with the use of digital media across the different life domains such as education, employment and interpersonal relationships (Carretero, Vuorikari, \& Punie, 2017). A competent digital media user is expected to be aware and to appropriately utilize occupational and/or educational innovations and possibilities accommodated by digital media (He \& Li, 2019). They are also expected to be able to effectively handle legal issues related to digital media use, as well as issues related to the validity and the reliability of information provided by it (Nouri, Zhang, Mannila, \& Norén, 2020). Digital competence has been a valuable resource during the COVID19 pandemic, when online presence and activities often substituted offline ones, acquiring a preponderant role in many individuals' lives (e.g., online education and online socializing have markedly increased; Hollis, Livingstone, \& Sonuga-Barke, 2020). In that line, He and Li (2019) found a significant link between digital competence and digital informal learning in both Belgian and Chinese students.

Digital acculturation is defined here as a correlate of digital competence. It describes the need of contemporary digital natives to concurrently develop competencies in effectively functioning both in their real and digital worlds, where interactions may entail different characteristics/features. For instance, cyberculture and cyber-relationships are interwoven with internet-related anonymity, accessibility, and affordability, the so-called 'Triple A Engine' (Cooper, Scherer \& Marcus, 2002). These factors are intrinsic to the way individuals conduct themselves online. Thus, an individual may say or do things online that they would never say or do face-to-face (Han, Geng, Jou, Gao, \& Yang, 2017; Keipi, 2018). The generalization of such behaviors offline may be dysfunctional. Digitally acculturated individuals should be able to maintain relationships in both contexts, understanding their different principles and ethics, while being able to code-switch between the real world and the cyber-world, as necessary. In that line, Sharabi (2020) investigated the prospects of romantic relationships that were initiated via digital dating apps employing algorithms to match users. Despite findings suggesting that algorithmic matching was ineffective, users' perception of its effectiveness was predictive of the first date outcome, indicating their reduced capacity to 
distinguish cyber-culture and real-world relationship demands (i. e. algorithmic matching success may not equal real-life relationships success).

Digital resilience, also considered as a digital media use adaptive outcome, involves two prerequisites. First, the digital media user, and/or the context/societal group involved with digital media use, should have encountered an important peril/hazard regarding their concurrent and future adaptation. Second, the individual's or system's operation and performance should be adequate in the context of this adversity (e.g., an individual's developmental tasks are met; Motti-Stefanidi \& Masten 2020; Reynolds \& Parker, 2018). It assumes a combination of resources such as critical thinking, technological literacy, skills in digital interactions, and digital safety, while it reinforces an individuals' or a systems' (i.e., family, community systems) ability to weather and resume challenges that may compromise their health and prospective evolution (see Livingstone \& Haddon, 2012). For instance, a natural disaster and/or significant political event/unrest affecting both individually, and at a group-level, online users, who might employ social media to more effectively manage/address it (Yuan, Li, Liu, Zhai, \& Qi, 2021). Thus, digital resilience is a dynamic concept, utilized across multiple levels, including digital media users and the real proximal and broader-distant context in which their lives are embedded.

Indicative evidence of digital resilience can be found in the work of Lavis and Winter (2020). They conducted an ethnographic study of digital interactions occurring on Twitter, Reddit and Instagram in relation to self-harm. They found that users received peer support online, helping them to better deal with their self-harming behaviors. For instance, an online protective factor contributing to youth's digital resilience could be the emergence of self-help digital groups, such as the Online Gamers Anonymous (olganon.org, 2020), where gamers, who identify as gaming problematically, aim to recover via peerorganized Zoom meetings, chatrooms, and forum interactions. Such digital self-help groups, which resemble those operating offline to address addictions (e.g., Alcoholics Anonymous; Sussman, 2010), have also appeared in relation to the excessive use of pornography. A popular example is NoFap on reddit 
(reddit.com/r/NoFap/, 2020), where participants share resources and set group goals to abstain from pornography/masturbation for progressively longer periods of time (Fernandez, Kuss \& Griffiths, 2020).

\section{Maladaptive outcomes related to digital media use}

Problematic Internet Use (PIU): This is broadly defined as an individual's inability to control their use of the internet, spending excessive amounts of time while online, leading them to distress and/or impairment in their everyday life (Anderson et al, 2001). Initially, such use is a pleasure-seeking behavior (e.g., an individual uses the internet to feel better). However, for some it can become a way for a user to escape distressing feelings. PIU behaviors refer to all types of internet applications that an individual may abuse. Some scholars have suggested that it possesses qualities of a behavioral addiction, as a user may be totally preoccupied with their internet use, use the medium to change the way they feel, experience irritability and/or frustration when not online, progressively need to extend their use to achieve the same level of gratification, are unable to abstain/reduce their use, and experiencing problems in their lives due to it (Griffiths, 2017). Other scholars conceive PIU primarily as an impulse-control disorder, due to being interwoven with significant impulse/inhibitory control deficits (Anderson et al., 2017). At least four different types of digital media applications (i.e., online gaming, online pornography, online gambling, and social media use) have been reported as likely high risk for PIU behaviors (Brand, Rumpf, King, Potenza, \& Wegmann, 2020; Taylor \& Gavey, 2020; Lopez-Gonzalez, Griffiths, \& Estévez, 2020; Sun \& Zhang, 2020).

In light of these, Internet Gaming Disorder (IGD), was included in the fifth edition of the Diagnostic and Statistical Manual of Mental Disorders (DSM-5; American Psychiatric Association, 2013) as a tentative condition. IGD has been defined as persistent engagement with games, either with internet or noninternet computerized games, leading to impairment. The World Health Organization (WHO) officially ratified Gaming Disorder (GD) as a diagnostic classification in May 2019. A meta-analysis of 16 studies estimated a $4.6 \%$ pooled prevalence of GD among adolescents, with males being at higher risk (Fam, 2018). Nevertheless, some scholars support abolishing addiction-like conceptualizations for excessive use of 
technology, as this has become an inherent component of contemporary life (Ellis, 2019; Panova \& Carbonell, 2018). They further argue that PIU presentations (e.g. excessive use of internet games, social media, online porn) may constitute the way pre-existing conditions exhibit on the internet domain (e.g., an individual is depressed, and they regulate their feelings through their online use; Ryding \& Kaye, 2017; Starcevic, Billieux \& Schimmenti, 2018).

\section{Framing digital media use in a multilevel developmental context}

The key goal of the CDF model is to explain why and how some digital natives do well with respect to age-related developmental tasks (Masten \& Motti-Stefanidi, 2020), while others may not or may do less well, when using digital media. In line with past recommendations, it takes into consideration individuals' experience within the digital context itself (Livingstone et al., 2018; Valkenburg's and Peter's, 2013). Johnson and Puplambou (2008) re-conceptualized techno-subsystem framework, enriched with elements from Douglas' internet addiction model (2008), provides the backbone for organizing the CDF sources of influences. Therefore, the techno-subsystem is acknowledged here as a cyber-layer/system involving a dynamic (rather than static) cyberworld/digital media world. Indeed, digital media literature has introduced the concepts of online presence, online flow, and virtual self-presence, to describe an individual's immersive experience of their digital context, activity, and self respectively (Antonaci, Klemke, Lataster, Kreijns, \& Specht, 2019; Chen et al., 2000; Lombard \& Jones, 2015; Ratan \& Hasler, 2010).

Accordingly, the definition of the technological subsystem is updated from a composition of hardware effects into a dynamic cyber-reality. In line with Johnson's and Publambou's (2008) initial suggestion, this operates as a separate level, system and/or context transactionally influencing an individual's development/behavior. Evidence considering the physiological outcomes of the interaction between the user and digital media has been summarized by Stiglic and Viner (2019). Their meta-review concluded that a range of risks, including compromised diet, mood, and quality of life, as well as adiposity may relate to one's screen time. 
CDF views an individual's behavior as perpetually changing across a spectrum of adaptive and maladaptive patterns. These occur on the basis of the interplay between push effects associated with characteristics of the users (e.g., an individual is depressed and aims to escape their feelings online) and their proximal and distal context (e.g., an individual's family or peer relationships are highly digitally engaged and social compliance effects push them online); and pull effects referring to features of the specific application (e.g., a leveled structured of online activity that generates a sense of challenge in internet games; Douglas et al., 2008). More specifically, moving from the center to the periphery of the model, the levels of influence considered include the users and their cyber-layer/system, followed by a sequence of real-life context influences (e.g., an individual's immediate and distant context). Therefore, the cyber-layer/system should be conceived as concurrent and interdependent with other sources of influences, such as an individual's family, school and their broader society (Livingstone \& Haddon, 2012). Online and offline contexts bi-directionally influence each other such that what constitutes an outcome at time 1, may become an effect at time 2, etc. Therefore, although users may initially be influenced by the cyber-layer/system, they also progressively exert an influence on it. Concurrently, parallel bi-directional associations occur between the cyber-layer and an individual's surroundings, as it affects an individual's family and even school via complex relationships that change over time.

Ballings and Van den Poel (2015) examined the increases in an individual's Facebook use time via the evaluation of algorithms that progressively tailor (via prediction models) users' interface to their specific profile. They compared the performance of various alternative algorithms (i. e. logistic regression, stochastic adaptive boosting, etc.) and concluded that such techniques can maximize usage (i.e. cyber-layer effecting the user). Indeed, such effects (i.e., tailoring the social media content to a user's profile to increase engagement) have been shown to reinforce polarization when considered at a broader group/societal level (i. e. cyber-layer/system being first affected and later affecting one's macro-system; Sîrbu, Pedreschi, Giannotti \& Kertész, 2019). Indicatively, Sîrbu and colleagues (2019) assumed that users' views can become progressively more rigid due to their selected exposure to digital media content. After amending 
the selection process to increase chances of like-minded individuals getting paired together online, they found an increased possibility of opinions fragmentation/polarization and a dramatically reduced chance of opinion convergence. They therefore suggested that an initial opinion fragmentation in a society predisposes such possibility, which is then digitally perpetuated by algorithmic biases in an upward spiral. These changes are reflected by a chrono-system upon which the rest of the systems and their interactions move over time. To address these issues, each of the CDF sources of influences and interactions are described below from the center to the periphery of the model (i.e., user, cyber-layer, real-context and chrono layers).

Figure 1. Cyber-Developmental Framework

\section{User related sources of influence}

To describe the influences related to the unique combination of characteristics of the digital media users regarding the outcomes of their digital media use, the CDF adopts Valkenburg and Peter's approach (2013).

Differential susceptibility to digital media describes how the exposure to the same digital media could generate unique and even opposite responses according to who the user is (Valkenburg \& Peter, 2013). This aligns with the 'rich get-richer' hypothesis suggesting that youth who are already doing well with respect to major developmental tasks, such as their relationship with peers or their romantic relationships, may use digital means because it provides them additional help. Thus, they strengthen their already existing developmental competence (Bouchillon, 2020). Nonetheless, it also supports the 'poor get poorer' hypothesis which proposes that youth with difficulties in the personal and interpersonal domains, may use the internet and its applications in a compensatory way (Kardefelt-Winther et al., 2017).

In this context, individuals' biological, psychological, personality, and socio-demographic traits are suggested to co-define the level of their differential susceptibility to digital media. Furthermore, some users might be more sensitive either to adverse or beneficial digital media behavioral effects (i.e., like an individual multiplier; Belsky \& Pluess, 2009). This also explains how the same factors, in this case digital 
media use, can produce different behavioral outcomes (developmental principle of multi-finality), while similar behavioral outcomes may be produced by different factors (developmental principle of equi-finality; Cicchetti \& Toth, 2009). For instance, Steinsbekk and colleagues (2021) illustrated the diversity of digital media use outcomes via assessing prospectively children from Norway. They suggested that social media use orientated towards others (e.g., consuming/ browsing others' newsfeed) progressively decreased females', but not males', self-esteem related to their appearance. Beyens and colleagues (2020) also showed that social media use differentially affects teens' wellbeing. Researchers examined the pattern of using social media to consume the material of others without self-expression/participation (e.g. browsing content, without making comments/posts). They concluded significant individual differences with approximately $40 \%$ of the users experiencing no effect, almost half of them experiencing improved feelings, while $10 \%$ deteriorating.

\section{Cyber-layer/system level of influence}

To describe the cyber-layer, the CDF emphasizes an individual's experience of their virtual context, virtual activity and virtual self. It also illustrates the significance of the potential interactions between these three elements, within the cyber-layer and across the other levels of the model, for understanding digital media use and abuse.

Virtual presence: Experiencing the digital environment as real, and feeling that one is actually there (and not in real life), is defined as presence [alternatively known as 'telepresence', 'mediated presence' or 'virtual presence' (Lombard \& Jones, 2015)]. Presence has been described as an immersive state that occurs when one ignores the mediating role of the technology when accessing a virtual environment (Lombard \& Jones, 2015). Initially the term was used to define an individual's transportation into a visualized digital environment. Nevertheless, it was quickly established that presence can be experienced across a range of digital contexts without necessitating a world resembling environment per se (Lombard \& Jones, 2015).

Different notions of presence have been proposed based on the medium employed. For instance, 'immersive virtual presence' and 'social robotic presence' occur when virtually immersive and robotic 
technological means are used respectively (Coradeschi, Loutfi, Kristoffersson, Cortellessa, \& Eklundh, 2011; Riva et al., 2014). Others have supported variations on presence based on the human senses involved. For example 'auditory' and 'haptic' presence describe respective states, where the senses of hearing and touching are consumed by the digital context (Witmer, Jerome \& Singer, 2005). Finally, based on the digital medium's capacity to accommodate interactions between users, the concepts of 'co-presence' and 'social presence' are occasionally employed (Nowak \& Biocca, 2003). Co-presence identifies the level that a user may be aware and experience others in the digital context (Antonaci et al., 2019). Social presence refers to the absorbing sense of a digital medium due to accommodating interactions between, and not just experiencing, users (Nowak \& Biocca, 2003; Biocca, Harms \& Burgoon, 2003).

Presence involves a range of different levels of intensity from minimum to maximum both between, as well as within individuals (Stavropoulos et al., 2019). Specifically, the extent that the users' mental world is occupied by digital material, and the level of attention/cognitive focus that this attracts, defines the intensity of presence they will experience (Lombard \& Jones, 2015). In that line, a pivotal factor reinforcing presence is a user's 'suspension of disbelief' (Lombard \& Jones, 2015). This reflects an individual's tendency to ignore evidence and thoughts, which feed distrust considering the real nature of digital contexts. How conscious and deliberate such behaviors may be, has been also said to vary. Nevertheless, it is hypothesized that high levels of presence (although a threshold is yet to be clarified) entertain an individual's sense of 'escape' from the real world (Stavropoulos et al., 2019).

The more realistic/naturalistic the features of the online content are (entailing both environment and activity), the higher the level of presence experienced tends to be (Lombard \& Jones, 2015; Shin, Song, Kim, \& Biocca, 2019). Such effects are additionally reinforced by the progressively higher number of augmented applications (Riener, Gabbard, \& Trivedi, 2019). Augmented or blended contexts are defined as digital environments that are augmented/enhanced by real-life elements (Milgram, Takemura, Utsumi, \& Kishino, 1995). A common augmented reality example refers to internet games with multiple players, where an individual can socialize and have transactions with real money and in real time (Anderson et al., 
2017). It is noted that there appears to be a continuum from reality to virtuality, with varying levels of augmented reality and augmented virtuality in between (Milgram et al., 1995; Hamari et al. 2019). The more integrated that digital and real elements become, the more meaningful in real terms the virtual context tends to be (Bourdon, 2020; Riener et al., 2019).

Online Flow: The level of user's engagement with their online activity has been also closely tied with one's affect (Stavropoulos et al., 2018). The concept of 'flow', as originally introduced in the context of positive psychology, was adapted to refer exclusively to online activities in the notion of 'online flow' (Csikszentmihalyi \& Nakamura, 2018; Stavropoulos et al., 2018).

Flow state is reached when an individual is totally consumed by their current activity (Csikszentmihalyi \& Nakamura, 2018). It is characterized by: (i) an elevated sense of physiological and psychological commitment with what an individual is doing; (ii) a complete allocation of attention; (iii) lack of consideration and care regarding the completion of the performed activity itself and; (iv) a distorted feeling of time and a sense that this is significantly accelerated. Flow is considered as an internal state of motivation that can exceed and get prioritized over physiological drives such as sleep and hunger (Csikszentmihalyi \& Nakamura, 2018).

Consequently, online flow is defined as being in an emotional state, that takes place while a user is engaged with an online activity, involving an uninterrupted succession of reactions to the medium. It is inherently gratifying and combined with a reduction (or even complete absence) of an individual's awareness/consciousness considering their offline context/activity (Stavropoulos et al., 2018). Therefore, it functions in a similar way to presence. However, while presence is attributed to the online context, online flow is caused by the user's activity within it (Polito \& Hitchens, 2020).

A flow state assumes a delicate pairing of activity-related challenges with an individual's level of competence (Stavropoulos et al., 2018). If an individual's perception of challenges related to an activity outweighs their relevant capacity (i.e., it is significantly more difficult than they can achieve), they feel disempowered, and demotivated, and then disengage. They also may disengage due to boredom and lack 
of interest if the reverse occurs (i.e., perceived challenges are significantly lower to an individual's skills). As an individual's online skills progressively increase due practice, their online challenges need to also increase. Activity demands need to constantly mildly exceed a user's perceived skills for them to remain invited/engaged (Csikszentmihalyi \& Nakamura, 2018). In the offline world, this can occur with a natural/organic selection of activities to the extent that these precipitate and perpetuate one's sense of flow (Csikszentmihalyi \& Nakamura, 2018). In online activity, this requires planning and structure (Stavropoulos et al., 2018). An application's challenges need to increase in such a calculated way that matches the expected rate of increase in a user's related competence. The latter needs to have been foreseen by the application developer (in game terms 'levelling up'; Stavropoulos et al., 2018).

Virtual self-presence: An individual's self-presence is also significant for the way they experience the digital world (Polito \& Hitchens, 2020). This often underpins differences between users' offline and online behaviors, prompting the introduction of the term 'virtual personality' (McCreery, Krach, Schrader, \& Boone, 2012; Yee, Harris, Jabon \& Bailenson, 2011). Character development features, embedded within online applications accommodate the expression of an individual's virtual personality, such as in online games and more recently in social networking sites. These enhance attractiveness, with a number of interactive applications moving towards integrating anthropomorphic digital impersonation ('avatars'; Liao, Cheng \& Teng, 2019 ; Messinger, Ge, Smirnov, Stroulia \& Lyons, 2019).

The word 'avatar' is a development of the Sanskrit word 'avatāra' referring to the transformation of Vishnu (a Hindi god) to earthly figures (Juul \& Klevjer, 2016). It indicates a perception of 'embodiment', employed primarily in online videogames, to describe the connection between the individual offline self and their in-game figure. The term is also used aside of online games to describe an individual's virtual self-presence, independent of the application of a human form (Nowak \& Fox, 2018). Therefore, understanding the user-avatar relationship (UAR) is deemed important (Korkeila, \& Hamari, 2020).

From a cyber-psychological perspective, the notion of 'self-presence' was initially conceived (in an analogy with that of presence) to describe the fusion of a user's psyche with their avatar. It describes how 
the users linked with their avatars physiologically and emotionally (Biocca, 1997). Prospectively, the connotation of self-presence changed to reflect the condition in which an individual's virtual-self mixes with their self-perception in reality, regarding their presentation, thoughts and feelings (Ratan \& Hasler, 2010). Accordingly, an individual's self-presence online is informed by 'proto', 'core', and 'extended' selfpresence dimensions (Ratan \& Hasler, 2010). These entail, bodily ('the avatar's body is my body'), emotional ('the avatar's feelings are my feelings'), and identity ('I am my avatar... Their story is my story') aspects, respectively.

The psychodynamic theorizing of the UAR also views the user-avatar link as bi-directional, from the user to the avatar and vice versa, and supports that gamers fuse with their avatar in four ways (Blinka, 2008). First, identification, 'I am who s/he (the avatar) is' (i.e., identity mixing). Second, immersion, 'His/her needs are my needs' and may often be prioritized to real-life needs. For instance, the avatar may need to participate in an action online and that may be deemed as more important to an individual's reallife obligations. Third, compensation, 'S/he is/has what I want to be/have but I don't'. Fourth, repression, 'the avatar can do things that I cannot do in real-life" (e.g., being promiscuous or violent). Finally, the Proteus Effect' $(\mathrm{PE})^{1}$, 'I start behaving like him/her in real life'. In a powerful way, the PE takes place when a user's offline demeanor and conduct somehow aligns with their online impersonation. For instance, an individual might feel more confident due to their high online popularity or a tall in-game persona (Yee \& Bailenson, 2007).

Aiming to examine in particular the neurobiological correlates underpinning the link between digital media users and their avatars, Lemenager and colleagues (2017) used a mixed-methods design. Three groups of 19 participants, each classified as disordered gamers, disordered social networking users, and control group were assessed via functional magnetic resonance imaging and psychometric scales. They found that disordered gamers experienced left brain hyper-activations while reflecting with their avatar which correlated with their disordered gaming behaviors. Highlighting also the connection between an individual's physiological responses to avatars' experiences, Joyal and colleagues (2018) assessed 24 adults 
combining quantitative electroencephalography (qEEG) and psychometric assessment. They observed that the pain experienced by an avatar prompted sensorimotor alpha wave suppression (SAS) in cortical areas, as in real life.

\section{Real-life context influences and time effects on digital media use}

The user and the cyber-layer/system are embedded with successively inclusive layers of context (Johnson \& Puplambou, 2008). Firstly, the user's micro-system entails immediate context effects such as an individual's family or school. Secondly, the meso-system refers to links between an individual's different micro-systems, such as the way their parents (i.e., family) may interact with their peers and school. This is embedded into the exo-system which describes environmental surroundings that may not directly affect the digital native, such as the infrastructure in their parents' employment space. Finally, the macrosystem refers to the influences related to an individual's broader community, such as cultural values or their stance towards digital media (Bronfebrenner \& Morris, 2007).

To illustrate differences in relation to one's country/macrosystem influences on their digital media use, Kardefelt-Winther et al. (2020) examined data of children and adolescents from Bulgaria, Chile, Ghana and the Philippines regarding their internet use and mental health. While in Bulgaria and Chile, higher reported internet use was weakly linked with an individual's wellbeing, this was not the case for Ghana and the Philippines where the association was insignificant. Similarly, Boer and colleagues (2020) studied the links between adolescents' wellbeing and their social media use in relation to their country of origin. Their findings showed that while in all countries, excessive social media (see Problematic Internet Use section) users reported reduced well-being, in countries where social media were more integrated to real social-life, they reported higher life satisfaction.

As a separate dimension in the space defined by the concentric contextual effects described, lies the chrono-system. This illustrates how the progress of time signifies transitions and changes on all the other layers of influence the CDF, as well as their interactions (Johnson \& Puplambou, 2008). For example, Teng et al. (2020) examined longitudinally Chinese students in relation to their disordered gaming 
behaviors and their attachments with their parents and peers. Their cross-lagged models showed that disordered gaming behaviors negatively linked with an individual's attachment with their peers and father, while they were also affected by an individual's peer attachment over time.

\section{Interaction effects related to digital media use}

All these sources (i.e., person attributes and successively embedded layers of cyber and real context) introduce influences for the digital native's development and adaptation. Therefore, their interplay defines an individual's level of digital resilience, digital competence, digital acculturation and/or PIU. Each different level accommodates hazards and/or resources, that may help them. The whole continuum of adaptive and maladaptive digital media use effects derives from the interplay of perils and benefits located across effects related to the individual and their cyber-world, family, school and society. Pull factors, attracting one online, are exclusively situated in an individual's cyber-layer, and may involve their experiences of presence, online flow and self-presence and their interplay. Similarly, push factors that encourage engagement with digital media are situated in all other context layers including the person. Stavropoulos et al. (2017) suggested that what may be a risk at the individual level, may well be a recourse at the proximal context level. They showed that while playing MMORPGs was a risk for the user, it turned into a protective effect when the gamer was located in a classroom with higher percentage of MMORPG players. They explained that gaming may indeed provide opportunities for real-life socialization between students.

Furthermore, and within the cyber-layer, the interplay between an individual's sense of virtual presence, flow, and self is also important. Pelet and colleagues (2017) examined the interplay between an individual's experience of their virtual context and activity. They analyzed online survey data and found that the user's sense of online presence associated with behaviors of enjoyment, concentration, challenge, control, and curiosity, which in turn was associated with online flow. Online flow was shown to link with an enhanced distortion of an individual's sense of time and increases in their social media consumption. The reverse relationship was investigated by Stavropoulos and colleagues (2013). They assessed Greek 
adolescents and reported that both presence and flow were associated with the level of excessive internet use behaviors exhibited. Their findings supported that higher sense of presence increased the association between a user's experience of online flow and their excessive internet use behaviors. Similarly, Polito and Hitchens (2020) found that interactive digital media use and increased engagement were both associated with higher experiences of presence, flow, and involvement with a virtual character. An individual's personality being more open to experience and more conscientious was shown to moderate such effects (Polito \& Hitchens, 2020, p.3).

Within this broad range of interactions, the digital world is affected itself, while continuing to affect an individual's development. Indicatively, Marciano, Schulz and Camerini (2020) examined how cyberbullying actors and receivers develop over time by conducting a meta-analysis. They suggested that cyberbullying and victimization experiences presented as both the causes and the results of offline bullying and victimization. They also noted that cyberbullying actors tended to present over time with more externalized problems (e.g., conduct difficulties), while receivers with more internalized problems (e.g., depression), with these associations being reciprocal.

In addition, the cyber-layer may also: (i) intersect with all other levels via augmented reality applications (i.e., digital applications involving real life elements such as space and money; Pombo \& Marques, 2020); (ii) mirror real life systems/communities (i.e., an individual's macro-system dynamics projected online via social media or within a game; e.g., users' in-game figures/ avatars against Trump movement in the online game second life; Cole, 2017) and; (iii) accommodate different ways of the user interacting with it, such as active or passive (Orben, 2020). For instance, when it comes to social media use, liking, messaging, and direct contact are considered active engagement, which has been shown to potentially support an individual's relationships (Orben, 2020). On the other hand, consuming photos, video, and newsfeed, which is considered passive engagement, was suggested to foster comparisons and jealousy, and therefore likely compromise an individual's well-being (Orben, 2020). 
The affective consequences of the intersection of the real and virtual world via augmented reality applications was evidenced by the work of de Gortari (2018). De Gortari defines "transfer phenomena" as unintentional/unplanned behaviors exhibited in relation to an individual's online activity. These entail online induced senses, perceptions, thoughts, and actions such as distorted experience of the real context when online or due to virtual influences (de Gortari, 2018). Their definition resembles 'priming', which refers to pre-conceptions due to exposure to media material (i.e., a negatively experienced online situation could underpin an aversive stance towards an associated real-life condition; Ash, 2016). Prompted by these, de Gortari (2018) assessed Pokémon Go gamers in relation to their experiences of immersion, augmented reality, and sound. They suggested that game transfer phenomena were more likely to be experienced when gaming was more intense. They also reported tactile hallucinations being more common, self-motion sensations being less reported, while such experiences being more common among location-based augmented digital games.

Similarly, at the individual level, the mirroring association between the real and virtual world may be best conceptualized by the notion of the 'digital phenotype' (Loi, 2019). Like real life behaviour, online behaviour can provide significant information about an individual's mental health and behaviour. As reduced appetite and sleep may provide the offline 'footprint' (i.e., a phenotype) of depression, an individual's Facebook posts (i.e., frequency, language) when analysed with pattern-recognition algorithms provide the online 'footprints' of depression and predict suicidal risk (Loi, 2019). Thorstad and Wolff (2019) used a Reddit Application Programming Interface (API) to access four clinically-focused reddit forums on ADHD, anxiety, bipolar disorder, and depression. They employed a language-based machine learning model (MLM; L2-penalized logistic regression via the Python library scikit-learn) to predict an individual's specific reddit forum membership. Their MLM predicted correctly the membership/distribution of previously unseen data at a level of accuracy exceeding $75 \%$. They also highlighted differentially predictive language for each of the symptoms examined. For instance, they 
suggested that depression is evidenced by the descending sequence of words sadness, life problems, drugs, health, worthlessness, ugliness, and harm.

The same cyber-layer mirroring association between an individual's distal contextual systems (e.g., exo-system and macro-system) may be evidenced by the work of Li et al. (2019). While focusing on the understanding of social perceptions concerning the use of supposedly more and less environmentally friendly energy sources, such as solar and coal, they conducted a big data mining experiment. They accrued tweets posts over a period of six months including relevant hashtags (e.g., \#coal, \#coalmine \#solarfarm, \#solar, \#solarpower). They then related the posts to USA regions using an individual's self-reported location available through Twitter meta-data. Their results reflected region-defined energy preferences with more traditional coal-mining areas being significantly more positive to coal compared to central coastal areas.

\section{Priorities, future directions, and conclusions}

The complexity of such effects and processes, as conceptualized in the cyber-developmental framework, can provide the basis for reframing the questions around the impact of digital media use on the development and mental health of contemporary digital natives. Therefore, the literature should move to more person targeted sequences of questions such as "who?", "when?", "where?”, "where digitally?”, "by doing what digitally?" and "by being who digitally?" can benefit or not. Such questions capture the range of the variability of individual differences in relation to the effects of digital media use, while concurrently maximizing the opportunities and minimizing the risks.

Such major opportunities may involve digital interventions for health (i.e., apps and online treatment programs; Ahmad, 2020). For instance, in which cases/conditions can 'serious games' be beneficial (Drummond, Hadchouel, \& Tesnière, 2017). These refer to 'gamified' interactive applications employing gaming challenges and enticement to equip users with skills and/or knowledge useful for real-life health concerns or practical tasks. For example, Depression Quest is an interactive fantasy game, with players 
assuming the life of somebody experiencing depression, while addressing every day challenges and managing their experience (Parkin, 2014).

Similarly, major risks should be approached by case-centered questions. For example, in which cases/conditions is one more likely to be at risk for the excessive use of digital games, social networking sites, online pornography and/or online gambling? Such questions are expected to shed more insight into disagreements between scholars considering the nature of these phenomena and how they should be effectively addressed (Ellis, Kaye, Wilcockson \& Ryding, 2017; Griffiths, 2017; Ryding Kaye 2017).

These should be attended by methodologically-enhanced research efforts. Such studies should involve the use of digital tracing data, alongside psychometric scales, followed by longitudinal and multilevel data collection in both the real and the digital world to allow more robust conclusions to be derived (Ellis, 2019). Recent studies have used such methodologies which benefit from the volume, the velocity, and the variability of digitally collected data or ecologically valid momentary assessments (e.g., keyboard kinematics/keystroke activity, emoji use patterns, voice activity detection algorithms, wearable actigraphy; Sano et al., 2018; Stasak et al., 2019; Van Dam et al., 2019). Transparency is required regarding the data collected, as well as the statistical processes employed to analyze it (Orben, Dienlin, \& Przybylski, 2019; Twenge, Haidt, Joiner \& Campbell, 2020). Finally, although individual differences should be considered, the effect of the digital world, as any other context surrounding the individual, should also be acknowledged.

Conclusively, a more careful consideration of the effects of internet and digital means use in the formation of an individual's behavior and identity is required in both scholarly and clinical work. This is necessitated by the rapid changes that dynamically converge the offline and the online contexts. Such convergence inevitably requires the merging of advancements of different fields of psychology, digital media, and developmental studies.

Note 1: Proteus was an ancient Greek god of transformations, who could present in various figures to avoid sharing his privileged knowledge of the past and the future. Given this capacity, his name was used to describe the transformative effects of an individual's online to offline behavior (Yee, 2007). 


\section{References}

Absolute Market Insights (2020). Online porn market 2019 2027. Retrieved December 18, 2020, from: https://www.absolutemarketsinsights.com/reports/Online-Porn-Market-2019-2027-551

Ahmad, A., Mueller, C., \& Tsamakis, K. (2020). Covid-19 pandemic: A public and global mental health opportunity for social transformation? BMJ, 369. doi: 10.1136/bmj.m1383

Anderson, E. L., Steen, E., \& Stavropoulos, V. (2017). Internet use and problematic internet use: A systematic review of longitudinal research trends in adolescence and emergent adulthood. International Journal of Adolescence and Youth, 22(4), 430-454. doi: 10.1080/02673843.2016.1227716

Antonaci, A., Klemke, R., Lataster, J., Kreijns, K., \& Specht, M. (2019, September). Gamification of MOOCs adopting social presence and sense of community to increase user's engagement: an experimental study. In: European Conference on Technology Enhanced Learning (pp. 172-186). Springer, Cham.

Ash, E. (2016). Priming or proteus effect? Examining the effects of avatar race on in-game behavior and post-play aggressive cognition and affect in video games. Games and Culture, 11(4), 422-440. doi: $10.1177 / 1555412014568870$

American Psychiatric Association (2013). Diagnostic and statistical manual of mental disorders (5 ${ }^{\text {th }}$ ed.). Arlington, VA: American Psychiatric Publishing. https://doi.org/10.1176/appi.books.9780890425596

Ballings, M., \& Van den Poel, D. (2015). CRM in social media: Predicting increases in Facebook usage frequency. European Journal of Operational Research, 244(1), 248-260. Doi: 10.1016/j.ejor.2015.01.001

Belsky, J., \& Pluess, M. (2009). Beyond diathesis stress: differential susceptibility to environmental influences. Psychological Bulletin, 135(6), 396-404. doi:10.1037/a0017376

Beyens, I., Pouwels, J. L., van Driel, I. I., Keijsers, L., \& Valkenburg, P. M. (2020). The effect of social media on well-being differs from adolescent to adolescent. Scientific Reports, 10(1), 1-11. Doi: 10.1038/s41598-020$67727-7$

Biocca, F. (1997). The cyborg's dilemma: Progressive embodiment in virtual environments. Journal of ComputerMediated Communication, 3(2), JCMC324. doi:10.1111/j.1083-6101.1997.tb00070.x

Biocca, F., Harms, C., \& Burgoon, J. K. (2003). Toward a more robust theory and measure of social presence: Review and suggested criteria. Presence: Teleoperators \& Virtual Environments, 12(5), 456-480. doi:10.1162/105474603322761270

Blinka, L. (2008). The Relationship of Players to Their Avatars in MMORPGs: Differences between Adolescents, Emerging Adults and Adults. Cyberpsychology: Journal of Psychosocial Research on Cyberspace, 2(1). Retrieved from https://cyberpsychology.eu/article/view/4211

Boer, M., van den Eijnden, R. J., Boniel-Nissim, M., Wong, S. L., Inchley, J. C., Badura, P., ... \& Stevens, G. W. (2020). Adolescents' intense and problematic social media use and their well-

Being in 29 countries. Journal of Adolescent Health, 66(6), S89-S99. Doi:

10.1016/j.jadohealth.2020.02.014 
Bouchillon, B. C. (2020). Social networking for interpersonal life: A competence-based approach to the rich get richer hypothesis. Social Science Computer Review. Doi: 10.1177/0894439320909506

Bourdon, J. (2020). From correspondence to computers: A theory of mediated presence in history. Communication Theory, 30(1), 64-83. doi: 10.1093/ct/qtz020

Brand, M., Rumpf, H. J., King, D. L., Potenza, M. N., \& Wegmann, E. (2020). Clarifying terminologies in research on gaming disorder and other addictive behaviors: Distinctions between core symptoms and underlying psychological processes. Current Opinion in Psychology. doi: 10.1016/j.copsyc.2020.04.006

Brand, M., Young, K. S., Laier, C., Wölfling, K., \& Potenza, M. N. (2016). Integrating psychological and neurobiological considerations regarding the development and maintenance of specific Internet-use disorders: An Interaction of Person-Affect-Cognition-Execution (I-PACE) model. Neuroscience \& Biobehavioral Reviews, 71, 252-266. doi: 10.1016/j.neubiorev.2016.08.033

Bronfenbrenner, U., \& Morris, P. A. (2007). The bioecological model of human development. Handbook of child psychology.

Carretero, S., Vuorikari, R., \& Punie, Y. (2017). The digital competence framework for citizens. Publications Office of the European Union.

Chang, C. T., Tu, C. S., \& Hajiyev, J. (2019). Integrating academic type of social media activity with perceived academic performance: A role of task-related and non-task-related compulsive Internet use. Computers \& Education, 139, 157-172. doi: 10.1016/j.compedu.2019.05.011

Chayko, M. (2020). Superconnected: The internet, digital media, and techno-social life. London: Sage Publications.

Cheng, C., \& Li, A. Y. L. (2014). Internet addiction prevalence and quality of (real) life: A meta-analysis of 31 nations across seven world regions. Cyberpsychology, Behavior, and Social Networking, 17(12), 755-760. doi: 10.1089/cyber.2014.0317

Csikszentmihalyi, M., \& Nakamura, J. (2018). Flow, altered states of consciousness, and human evolution. Journal of Consciousness Studies, 25(11-12), 102-114. Doi:

Cicchetti, D., \& Toth, S. L. (2009). The past achievements and future promises of developmental psychopathology: The coming of age of a discipline. Journal of Child Psychology and Psychiatry, 50(1-2), 16-25. doi: 10.1111/j.1469-7610.2008.01979.x

Cinelli, M., Quattrociocchi, W., Galeazzi, A., Valensise, C. M., Brugnoli, E., Schmidt, A. L., ... \& Scala, A. (2020). The Covid-19 social media infodemic. arXiv, 2003.05004.

Coldwell, D. (2019). Negative influences of the 4th industrial revolution on the workplace: Towards a theoretical model of entropic citizen behavior in toxic organizations. International Journal of Environmental Research and Public Health, 16(15), 2670. doi: 10.3390/ijerph16152670

Cooper, A., Scherer, C., \& Marcus, I. D. (2002). Harnessing the power of the internet to improve sexual relationships. Sex and the Internet: A guidebook for clinicians, 209-30. Btunner-Routledge. New York. 
Coradeschi, S., Loutfi, A., Kristoffersson, A., Cortellessa, G., \& Eklundh, K. S. (2011). Social robotic telepresence. Paper presented at the 2011 6th ACM/IEEE International Conference on Human-Robot Interaction (HRI), Boston, Massachusetts, USA.

Davis, R. A. (2001). A cognitive-behavioral model of pathological Internet use. Computers in Human Behavior, 17(2), 187-195. doi: 10.1016/S0747-5632(00)00041-8

De Gortari, A. B. O. (2018). Empirical study on game transfer phenomena in a location-based augmented reality game. Telematics and Informatics, 35(2), 382-396. Doi: 10.1016/j.tele.2017.12.015

Dong, G., \& Potenza, M. N. (2016). Risk-taking and risky decision-making in Internet gaming disorder: Implications regarding online gaming in the setting of negative consequences. Journal of Psychiatric Research, 73, 1-8. doi: 10.1016/j.jpsychires.2015.11.011

Douglas, A. C., Mills, J. E., Niang, M., Stepchenkova, S., Byun, S., Ruffini, C., ... \& Blanton, M. (2008). Internet addiction: Meta-synthesis of qualitative research for the decade 1996-2006. Computers in Human Behavior, 24(6), 3027-3044. doi: 10.1016/j.chb.2008.05.009

Drummond, D., Hadchouel, A., \& Tesnière, A. (2017). Serious games for health: three steps forwards. Advances in Simulation, 2(1), 3. doi: 10.1186/s41077-017-0036-3

Ducheneaut, N., Yee, N., Nickell, E., \& Moore, R. J. (2006). Building an MMO with mass appeal: A look at gameplay in World of Warcraft. Games and Culture, 1(4), 281-317. Doi: 10.1177/1555412006292613

Ellis, D. A., Kaye, L. K., Wilcockson, T. D., \& Ryding, F. C. (2018). Digital traces of behaviour within addiction: Response to Griffiths (2017). International Journal of Mental Health and Addiction, 16(1), 240-245. Doi: $10.1007 / \mathrm{s} 11469-017-9855-7$

Eurostat (2020-07-01). Being young in Europe today - digital world. Europa.EU. Retrieved December 18, 2020,


_digital_world

Fam, J. Y. (2018). Prevalence of internet gaming disorder in adolescents: A meta-analysis across three decades. Scandinavian journal of psychology, 59(5), 524-531. doi: 10.1111/sjop.12459

Fernandez, D., Kuss, D.J. \& Griffiths, M.D. (2021). The pornography 'rebooting' experience: A qualitative analysis of abstinence journals on an online pornography abstinence forum. Archives of Sexual Behavior, in press.

Gomes, A., \& Dias, J. G. (2019). Internet usage patterns: Segmentation of European users using a multilevel latent class model. In: Program and book of Abstracts XXVI Meeting of the Portuguese Association for Classification and Data Analysis (CLAD) (p. 67).

Gough, C. (2020-07-24). Genre breakdown of U.S. video game sales in 2018. Statista. Retrieved December 18, 2020, from: https://www.statista.com/statistics/189592/breakdown-of-us-video-game-sales-2009-by-genre/

Hamari, J., Malik, A., Koski, J., \& Johri, A. (2019). Uses and gratifications of pokémon go: Why do people play mobile location-based augmented reality games? International Journal of Human-Computer Interaction, 35(9), 804-819. Doi: 10.1080/10447318.2018.1497115 
Han, L., Geng, J., Jou, M., Gao, F., \& Yang, H. (2017). Relationship between shyness and mobile phone addiction in Chinese young adults: Mediating roles of self-control and attachment anxiety. Computers in Human Behavior, 76, 363-371. Doi: 10.1016/j.chb.2017.07.036

He, T., \& Li, S. (2019). A comparative study of digital informal learning: The effects of digital competence and technology expectancy. British Journal of Educational Technology,50(4), 1744-1758. Doi: 10.1111/bjet.12778

Hernandez, K., \& Roberts, T. (2018). Leaving no one behind in a digital world. Retrieved December 18, 2020, from: https://opendocs.ids.ac.uk/opendocs/handle/20.500.12413/14147

Hollis, C., Livingstone, S., \& Sonuga-Barke, E. (2020). The role of digital technology in children and young people's mental health-a triple-edged sword? The Journal of Clinical Psychology and Psychiatry. Doi: $10.1111 /$ jcpp. 13302

International Telecommunications Union (2017). ICT facts and figures 2017. Retrieved December 18, 2020, from: https://www.itu.int/en/ITU-D/Statistics/Documents/facts/ICTFactsFigures2017.pdf

Jackson, S., \& Goossens, L. (2020). Handbook of adolescent development: London: Psychology Press.

Johnson, G. M., \& Puplampu, K. P. (2008). Internet use during childhood and the ecological technosubsystem. Canadian Journal of Learning and Technology/La Revue Canadienne de l'Apprentissage et de la Technologie, 34(1). Doi:10.21432/T2CP4T

Jones, C., Scholes, L., Johnson, D., Katsikitis, M., \& Carras, M. C. (2014). Gaming well: links between videogames and flourishing mental health. Frontiers in Psychology, 5, 260. doi: 10.3389/fpsyg.2014.00260

Joyal, C. C., Neveu, S. M., Boukhalfi, T., Jackson, P. L., \& Renaud, P. (2018). Suppression of sensorimotor

Alpha power associated with pain expressed by an avatar: a preliminary EEG study. Frontiers in Human Neuroscience, 12, 273. Doi: 10.3389/fnhum.2018.00273

Juul, J., \& Klevjer, R. (2016). Avatar. The International Encyclopedia of Communication Theory and Philosophy, 15.

Kardefelt-Winther, D., Heeren, A., Schimmenti, A., van Rooij, A., Maurage, P., Carras, M., ... \& Billieux, J. (2017). How can we conceptualize behavioural addiction without pathologizing common behaviours? Addiction, 112(10), 1709-1715. doi:10.1111/add.13763

Keipi, T. (2018). Relatedness online: An analysis of youth narratives concerning the effects of internet anonymity. Young, 26(2), 91-107. doi:10.1177/1103308817715142

Kemp, S. (2019). Digital trends 2019: Every single stat you need to know about the internet. The Next Web. Retrieved December 18, 2020, from: https:/thenextweb.com/contributors/2019/01/30/digital-trends-2019-everysingle-stat-you-need-to-know-about-the-internet/

Kesharwani, A. (2020). Do (how) digital natives adopt a new technology differently than digital immigrants? A longitudinal study. Information \& Management, 57(2), 103170. doi: 10.1016/j.im.2019.103170 
Korkeila, H., \& Hamari, J. (2020). Avatar capital: The relationships between player orientation and their avatar's social, symbolic, economic and cultural capital. Computers in Human Behavior, 102, 14-21. Doi: 10.1016/j.chb.2019.07.036

Lavis, A., \& Winter, R. (2020). \#Online harms or benefits? An ethnographic analysis of the positives and negatives of peer-support around self-harm on social media. Journal of Child Psychology and Psychiatry. Doi: $10.1111 /$ jcpp. 13245

Leménager, T., Dieter, J., Hill, H., Mann, K., \& Kiefer, F. (2017). Internet addiction and the virtual selfimage. European Psychiatry, 41(S1), S26-S26. doi: 10.1016/j.eurpsy.2017.01.136

Lewczuk, K., Wojcik, A., \& Gola, M. (2019). Increase in the prevalence of online pornography use-objective data analysis from the period between 2004 and 2016 in Poland.

Liao, G. Y., Cheng, T. C. E., \& Teng, C. I. (2019). How do avatar attractiveness and customization impact online gamers' flow and loyalty? Internet Research. Doi: 10.1108/IntR-11-2017-0463

Litsou, K., Byron, P., McKee, A., \& Ingham, R. (2020). Learning from pornography: Results of a mixed methods systematic review. Sex Education, 1-17. doi:10.1080/14681811.2020.1786362

Livingstone, S., \& Haddon, L. (2012). Theoretical framework for children's internet use. Children, risk and safety on the internet: Research and policy challenges in comparative perspective, 1-14.

Livingstone, S., Mascheroni, G., \& Staksrud, E. (2018). European research on children's internet use: Assessing the past and anticipating the future. New Media \& Society, 20(3), 1103-1122. doi: 10.1111/jcpp.13302

Loi, M. (2019). The digital phenotype: A philosophical and ethical exploration. Philosophy \& Technology, 32(1), 155-171. doi: 10.1007/s13347-018-0319-1

Lombard M., Jones M.T. (2015) Defining Presence. In: Lombard M., Biocca F., Freeman J., IJsselsteijn W., Schaevitz R. (eds) Immersed in Media. Springer, Cham. https://doi.org/10.1007/978-3-319-10190-3_2

Lopez-Gonzalez, H., Griffiths, M. D., \& Estévez, A. (2020). Why some sports bettors think gambling addiction prevented them from becoming winners? A qualitative approach to understanding the role of knowledge in sports betting products. Journal of Gambling Studies. doi: 10.1007/s10899-020-09944-3

Lorenz, J. (2007). Continuous opinion dynamics under bounded confidence: A survey. International Journal of Modern Physics C, 18(12), 1819-1838.

Marciano, L., Schulz, P. J., \& Camerini, A. L. (2020). Cyberbullying perpetration and victimization in youth: a=A meta-analysis of longitudinal studies. Journal of Computer-Mediated Communication, 25(2), 163-181. Doi: 10.1093/jcmc/zmz031

Masten, A. S., \& Motti-Stefanidi, F. (2020). Multisystem resilience for children and youth in disaster: Reflections in the context of COVID-19. Adversity and Resilience Science, 1(2), 95-106. doi: 10.1007/s42844-020-00010$\mathrm{W}$

McCreery, M. P., Krach, S. K., Schrader, P. G., \& Boone, R. (2012). Defining the virtual self: Personality, behavior, and the psychology of embodiment. Computers in Human Behavior, 28(3), 976-983. Doi: 10.1016/j.chb.2011.12.019 
Messinger, P. R., Ge, X., Smirnov, K., Stroulia, E., \& Lyons, K. (2019). Reflections of the extended self: Visual selfrepresentation in avatar-mediated environments. Journal of Business Research, 100, 531-546. Doi: 10.1016/j.jbusres.2018.12.020

Milgram, P., Takemura, H., Utsumi, A., \& Kishino, F. (1995). Augmented reality: A class of displays on the realityvirtuality continuum. In: Telemanipulator and telepresence technologies (Vol. 2351, pp. 282-292): International Society for Optics and Photonics.

Nouri, J., Zhang, L., Mannila, L., \& Norén, E. (2020). Development of computational thinking, digital competence and 21st century skills when learning programming in K-9. Education Inquiry, 11(1), 1-17. Doi: $10.1080 / 20004508.2019 .1627844$

Nowak, K. L., \& Biocca, F. (2003). The effect of the agency and anthropomorphism on users' sense of telepresence, copresence, and social presence in virtual environments. Presence: Teleoperators \& Virtual Environments, 12(5), 481-494. doi:10.1162/105474603322761289

Nowak, K. L., \& Fox, J. (2018). Avatars and computer-mediated communication: A review of the definitions, uses, and effects of digital representations. Review of Communication Research,6, 30-53. doi: 10.12840/issn.2255-4165.2018.06.01.015

Odgers, C.L., \& Jensen, M.R. (2020). Annual research review: Adolescent mental health in the digital age: Facts, fears, and future directions. Journal of Child Psychology and Psychiatry, 61, 336-348. doi: 10.1111/jcpp.13219

Oleksy, T., \& Wnuk, A. (2017). Catch them all and increase your place attachment! The role of location-based augmented reality games in changing people-place relations. Computers in Human Behavior, 76, 3-8. Doi: 10.1016/j.chb.2017.06.008

Orben, A. (2020). Teenagers, screens and social media: A narrative review of reviews and key studies. Social Psychiatry and Psychiatric Epidemiology, 1-8. doi: 10.1007/s00127-019-01825-4

Orben, A., Dienlin, T., \& Przybylski, A. K. (2019). Reply to Foster and Jackson: Open scientific practices are the way forward for social media effects research. Proceedings of the National Academy of Sciences, 116(31). Doi: 10.1073/pnas. 1909553116

Panova, T., \& Carbonell, X. (2018). Is smartphone addiction really an addiction? Journal of Behavioral Addictions, 7(2), 252-259.

Parkin, S. (2014). Zoe Quinn's depression quest. The New Yorker, 9.

Pelet, J. É., Ettis, S., \& Cowart, K. (2017). Optimal experience of flow enhanced by telepresence: Evidence from social media use. Information \& Management, 54(1), 115-128. Doi: 10.1016/j.im.2016.05.001

Pew Research Center (2020-05-08). American trends panel wave 56. Pew Research Center. Retrieved December 18, 2020, from: https://www.pewresearch.org/internet/dataset/american-trends-panel-wave-56/

Pew Research Center (2019-07-12). Internet/broadband fact sheet. Pew Research Center. Retrieved December 18, 2020, from: https://www.pewresearch.org/internet/fact-sheet/internet-broadband/ 
Pew Research Center (2018-05-31). Teens, social media \& technology 2018. Pew Research Center. Retrieved December 18，2020， from: https://www.pewresearch.org/internet/2018/05/31/teens-social-mediatechnology-2018/

Polito, V., \& Hitchens, M. (2020). Digital media impacts multiple aspects of self-representation: An investigation of flow, agency, presence, character identification, and time perception. Psychology of Popular Media. Doi: 10.1037/ppm0000330

Pombo, L., \& Marques, M. M. (2020). The potential educational value of mobile augmented reality games: The case of EduPARK App. Education Sciences, 10(10), 287.

Prensky, M. (2009). H. sapiens digital: From digital immigrants and digital natives to digital wisdom. Innovate: journal of online education, 5(3). Doi:

Ratan, R., \& Hasler, B. S. (2010). Exploring self-presence in collaborative virtual teams. PsychNology Journal, 8(1). Doi:

Reynolds, L., \& Parker, L. (2018). Digital resilience: Stronger citizens online. London: Institute for Strategic Dialogue:

Riener, A., Gabbard, J., \& Trivedi, M. (2019). Special issue of presence: virtual and augmented reality virtual and augmented reality for autonomous driving and intelligent vehicles: guest editors' introduction. In: MIT Press. doi: 10.1162/pres_e_00323

Riva, G., Mantovani, F., \& Bouchard, S. (2014). Presence. In: B. K. Wiederhold \& S. Bouchard (Eds.), Series in anxiety and related disorders. Advances in virtual reality and anxiety disorders (p. 9-33: Springer Science + Business Media.

Ryding, F. C., \& Kaye, L. K. (2018). "Internet addiction": A conceptual minefield. International Journal of Mental Health and Addiction, 16(1), 225-232. Doi: 10.1007/s11469-017-9811-6

Saffo, D., Yildirim, C., Di Bartolomeo, S., \& Dunne, C. (2020, April). Crowdsourcing Virtual Reality Experiments using VRChat. In Extended Abstracts of the 2020 CHI Conference on Human Factors in Computing Systems (pp. 1-8).

Schoenherr, S. E. (2004). The Digital Revolution. Retrieved January $27^{\text {th }}$, 2021, from https://web.archive.org/web/20081007132355/http:/history.sandiego.edu/gen/recording/digital. $\mathrm{Html}$

Sharabi, L. L. (2020). Exploring how beliefs about algorithms shape (offline) success in online dating: A two-wave longitudinal investigation. Communication Research, 1-22. Doi: 10.1177/0093650219896936

Shin, M., Song, S. W., Kim, S. J., \& Biocca, F. (2019). The effects of 3D sound in a 360-degree live concert video on social presence, parasocial interaction, enjoyment, and intent of financial supportive action. International Journal of Human-Computer Studies, 126, 81-93. doi: 10.1016/j.ijhcs.2019.02.001

Sîrbu, A., Pedreschi, D., Giannotti, F., \& Kertész, J. (2019). Algorithmic bias amplifies opinion fragmentation and polarization: A bounded confidence model. PloS One, 14(3). Doi: 10.1371/journal.pone.0213246

Smith, Richard (2013). "What is digital media?". The Centre for Digital Media. Retrieved December 18, 2020, from: https://thecdm.ca/news/what-is-digital-media 
Snodgrass, J. G., Bagwell, A., Patry, J. M., Dengah II, H. F., Smarr-Foster, C., Van Oostenburg, M., \& Lacy, M. G. (2018). The partial truths of compensatory and poor-get-poorer internet use theories: More highly involved videogame players experience greater psychosocial benefits. Computers in Human Behavior, 78, 10-25. doi: 10.1016/j.chb.2017.09.020

Stavropoulos, V., Burleigh, T. L., Beard, C. L., Gomez, R., \& Griffiths, M. D. (2019). Being there: A preliminary study examining the role of presence in internet gaming disorder. International Journal of Mental Health and Addiction, 17(4), 880-890. doi: 10.1007/s11469-018-9891-y

Stavropoulos, V., Alexandraki, K., \& Motti-Stefanidi, F. (2013). Flow and telepresence contributing to Internet abuse: Differences according to gender and age. Computers in Human Behavior, 29(5), 1941-1948. doi: 10.1016/j.chb.2013.03.01

Stavropoulos, V., Griffiths, M. D., Burleigh, T. L., Kuss, D. J., Doh, Y. Y., \& Gomez, R. (2018). Flow on the Internet: a longitudinal study of Internet addiction symptoms during adolescence. Behaviour \& Information Technology, 37(2), 159-172. doi: 10.1080/0144929X.2018.1424937

Stavropoulos, V., Kuss, D. J., Griffiths, M. D., Wilson, P., \& Motti-Stefanidi, F. (2017). MMORPG gaming and hostility predict Internet addiction symptoms in adolescents: An empirical multilevel longitudinal study. Addictive Behaviors, 64, 294-300. doi: 10.1016/j.addbeh.2015.09.001

Steinsbekk, S., Wichstrøm, L., Stenseng, F., Nesi, J., Hygen, B. W., \& Skalická, V. The impact of social media use on appearance self-esteem from childhood to adolescence-A 3-wave community study. Computers in Human Behavior, 114, 106528. Doi: 10.1016/j.chb.2020.106528

Stiglic, N., \& Viner, R. M. (2019). Effects of screentime on the health and well-being of children and adolescents: A systematic review of reviews. BMJ Open, 9(1). Doi: 10.1136/bmjopen-2018-023191

Sun, Y., \& Zhang, Y. (2020). A review of theories and models applied in studies of social media addiction and implications for future research. Addictive Behaviors, 114. Doi: 10.1016/j.addbeh.2020.106699

Sussman, S. (2010). A review of Alcoholics Anonymous/Narcotics Anonymous programs for teens. Evaluation \& The Health Professions, 33(1), 26-55. Doi: 10.1177/0163278709356186

Symonds, J., Schoon, I., Eccles, J., \& Salmela-Aro, K. (2019). The development of motivation and amotivation to study and work across age-graded transitions in adolescence and young adulthood. Journal of Youth and Adolescence, 48(6), 1131-1145. doi: 10.1007/s10964-019-01003-4

Syngene Research (2019). Global e-learning market analysis 2019. Retrieved December 18, 2020, from: https://www.researchandmarkets.com/reports/4769385/global-e-learning-market-analysis-

Taylor, K., \& Gavey, N. (2020). Pornography addiction and the perimeters of acceptable pornography viewing. Sexualities, 23(5-6), 876-897. doi: 10.1177/1363460719861826

Teng, Z., Griffiths, M. D., Nie, Q., Xiang, G., \& Guo, C. (2020). Parent-adolescent attachment and peer attachment associated with internet gaming disorder: A longitudinal study of first-year undergraduate students. Journal of Behavioral Addictions, 9(1), 116-128. Doi; 10.1556/2006.2020.00011

Thorstad, R., \& Wolff, P. (2019). Predicting future mental illness from social media: A big-data approach. Behavior Research Methods, 51(4), 1586-1600. Doi: 10.3758/s13428-019-01235-z 
Twenge, J. M., Haidt, J., Joiner, T. E., \& Campbell, W. K. (2020). Underestimating digital media harm. Nature Human Behaviour, 4(4), 346-348. Doi: 10.1038/s41562-020-0839-4

Valkenburg, P. M., \& Peter, J. (2013). The differential susceptibility to media effects model. Journal of Communication, 63(2), 221-243. doi:10.1111/jcom.12024

WePC (2020). Video game industry statistics in 2020. WePC. Retrieved December 18, 2020, from: https://www.wepc.com/news/video-game-statistics/

Westlake, E. J. (2020). If you see me collapse, pause my strava. Sporting Performances: Politics in Play, 74.

World Health Organization (2019). Addictive Behaviours: Gaming Disorder. Retrieved December 18, 2020, from: http://www.who.int/features/qa/gaming-disorder/en/

Witmer, B. G., Jerome, C. J., \& Singer, M. J. (2005). The factor structure of the presence questionnaire. Presence: Teleoperators \& Virtual Environments, 14(3), 298-312. doi: 10.1162/105474605323384654

Xu, X., Wang, J., Peng, H., \& Wu, R. (2019). Prediction of academic performance associated with internet usage behaviors using machine learning algorithms. Computers in Human Behavior, 98, 166-173. doi: 10.1016/j.chb.2019.04.015

Yee, N., \& Bailenson, J. J. H. (2007). The Proteus effect: The effect of transformed self-representation on behavior. Human Communication Research 33(3), 271-290. doi:10.1111/j.1468-2958.2007.00299.x

Yee, N., Harris, H., Jabon, M., \& Bailenson, J. N. (2011). The expression of personality in virtual worlds. Social Psychological and Personality Science, 2(1), 5-12. doi: 10.1177/1948550610379056

Yuan, F., Li, M., Liu, R., Zhai, W., \& Qi, B. (2021). Social media for enhanced understanding of disaster resilience during Hurricane Florence. International Journal of Information Management, 57, 102289. Doi: 10.1016/j.ijinfomgt.2020.102289 




